\title{
Impact of single point mutations on the excitonic structure and dynamics in FMO complex
}

\author{
Anton Khmelnitskiy ${ }^{1}$ and Ryszard Jankowiak ${ }^{1,2}$ \\ ${ }^{1}$ Department of Chemistry, Kansas State University, Manhattan, KS, USA \\ ${ }^{2}$ Department of Physics, Kansas State University, Manhattan, KS, USA
}

The Fenna-Matthews-Olson (FMO) antenna complex from the green sulfur bacterium Chlorobaculum (C.) tepidum is an important model protein to study exciton dynamics and excitation energy transfer in photosynthetic complexes. Single point mutations provide an excellent opportunity for the study of exciton structure and interexciton level relaxation. In this bacterium, the energy harvested by chlorosomes is funnelled via the baseplate pigments and FMO trimers to the reaction center $(\mathrm{RC})$, where photochemistry takes place. The structure of the FMO protein from C. tepidum was solved and revealed a homotrimeric complex containing eight bacteriochlorophylls (BChl) $a$ per monomer [1].

In this work we focus on two mutants: 1) V152N (here mutation occurs near BChl 1, i.e. valine is replaced by asparagine); and 2) W184F (where tryptophan 184 near BChl 6 is replaced with phenylalanine). In order to obtain information on the excited state lifetimes $\left(T_{1}\right)$ and interexciton level relaxation in the mutants we measured low temperature absorption/emission spectra and performed high-resolution resonant HB experiments. It is well known that the excited state lifetimes, that are related to the so-called homogeneously broadened lines $\left(\Gamma_{\text {hom }}\right)$, are easily obtained via hole-burning spectroscopy as $1 / T_{2}=\left(\pi \mathrm{c} \Gamma_{\text {hom }}\right)^{-1}$ $=\left(\pi T_{1}\right)^{-1}+\left(\pi T_{2}{ }^{*}\right)^{-1}$, where $T_{2}$ is the total dephasing time and $T_{2}{ }^{*}$ is the pure dephasing time;

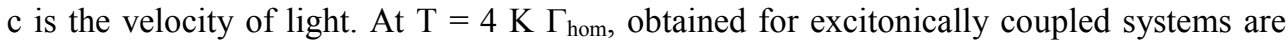
strongly dominated by the exciton level decay times $\left(T_{1}\right) . T_{1}=\left(2 \pi \mathrm{c} \Gamma_{\mathrm{hom}}\right)^{-1}$, where $\Gamma_{\text {hom }}=1 / 2 \Gamma_{\mathrm{ZPH}}$. In the modeling studies we apply a recently developed algorithm [2] to simultaneously fit multiple optical spectra. Intra-monomer interactions are modeled using non-Markovian Redfield theory. Calculations are performed for the FMO trimer.

Fig. 1 compares the extracted resonant holes (four black narrow spikes) obtained for the typically studied wild type (WT) FMO with holes burned in the V152N mutant (Frame A; ten red holes) and W16F mutant (Frame C; eleven red holes). Spectra a (black) and b (red) are the absorption spectra obtained at $4 \mathrm{~K}$ for typical WT FMO, V152N (Frame A) and W16F (Frame C) mutant samples respectively. All resonant holes in Fig. 1 are obtained with a constant fluence $\mathrm{f}=6 \mathrm{~J} / \mathrm{cm}^{2}$.

The absence of resonant bleach in WT FMO sample at $v_{\mathrm{B}} \geq 12450 \mathrm{~cm}^{-1}$, as illustrated in Fig. 1A/B, implies very fast (femtosecond) energy relaxation in this spectral range in agreement with ultrafast pump-probe and 2DES data [3]. That is, the lifetimes of the states excited in this region are too short to allow for an observable resonant bleach (broad ZPH). Likewise, the presence of ZPHs at higher energies in V152N (Fig. 1A) and W16F (Fig. 1C) indicates to slower ( $\mathrm{ps}$ ) energy relaxation for higher energy excitons compared to WT FMO. The slower experimentally estimated $T_{1}$ values for $\mathrm{V} 152 \mathrm{~N}(\sim 1 \mathrm{ps})$ and $\mathrm{W} 184 \mathrm{~F}(1.2-1.9 \mathrm{ps})$ 
mutants for high energy excitons are also in good agreement with the averaged calculated values of 1.1 and $1.3 \mathrm{ps}$, respectively. Figure 2 shows experimental/calculated absorbance $\left(\mathrm{a} / \mathrm{a}^{\prime}\right)$, emission $\left(\mathrm{b} / \mathrm{b}^{\prime}\right)$ and nonresonant hole-burned $\left(\mathrm{c} / \mathrm{c}^{\prime}\right)$ spectra for V152N mutant.
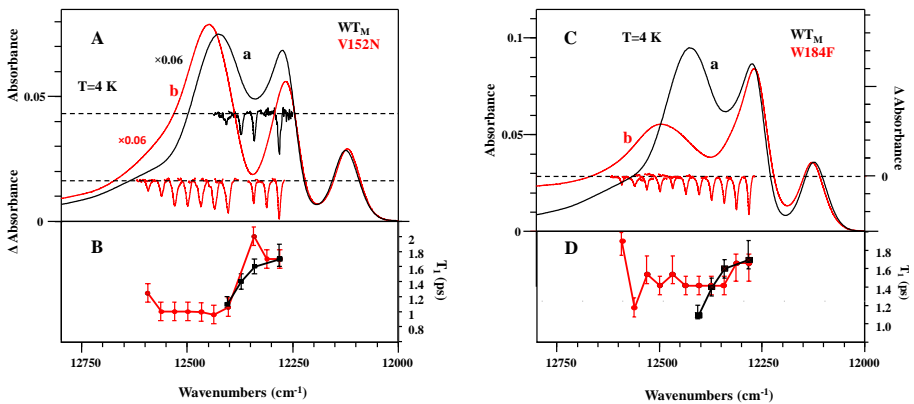

Fig. 1. Spectra a and $b$ are the $4 \mathrm{~K}$ absorption spectra obtained for a WT FMO (black curve a), V152N mutant (Frame A: red; curve b) and W184F mutant (Frame C: red; curve b). The top four holes (Frame A: black curves) and the red holes (at the bottom) correspond to the extracted resonant holes observed in a typical WT FMO, V152N mutant (Frame A) and W184F mutant (Frame C) respectively. The excited-state decay times $\left(T_{1}\right)$ calculated from hole widths $\left(\Gamma_{\mathrm{ZPH}}\right)$ obtained as a function of burn frequency $\left(v_{B}\right)$ for WT (black squares), V152N mutant (Frame B: red circles) and W16F mutant (Frame D: red squares) with corresponding error values (bars).
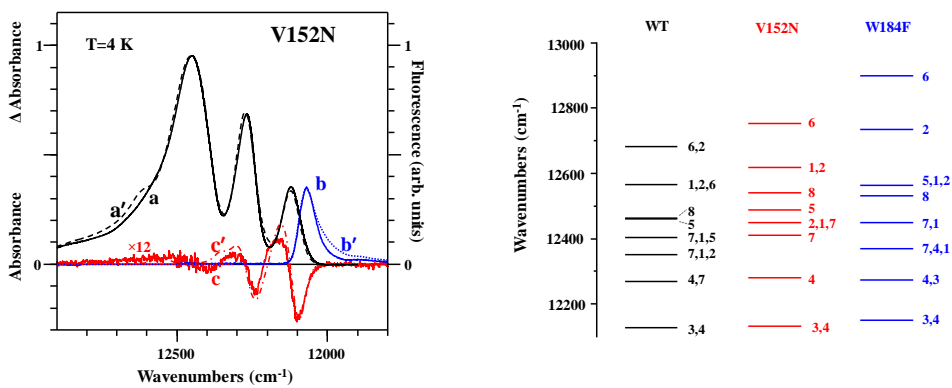

Fig. 2. Left frame: Black spectra are the experimental/simulated (solid/dashed curves a/a'). Blue curves show the experimental/simulated (solid/dashed curve $\mathrm{b} / \mathrm{b}^{\prime}$ ). Red curves $\mathrm{c}$ and $\mathrm{c}^{\prime}$ correspond to the experimental (solid) and calculated (dashed-dotted) nonresonant HB spectra; $v_{\mathrm{ex}}=20,490 \mathrm{~cm}^{-1}$ for V152N FMO mutant at $\mathrm{T}=4 \mathrm{~K}$. Right frame: exciton energy level scheme for monomers of WT and three mutants based on our calculations. Dominant pigment contributions $(>15 \%)$ for each exciton are indicated by corresponding $\mathrm{BChl}$ numbers.

Figure 2 (right frame) shows calculated exciton energy level scheme for WT and the two mutants. It is evident that mutations near BChl 1 and 6 result in red shift of higher energy excitons. Thus, we suggest that the site-energy shifts of the BChls 1 and 6 in particular, shared by several high-energy excitons, are responsible for the observed changes in higher energy excited-state decay times. As a result, those states shift to higher energies and overlap between them decreases. Moreover, longer excited-state decay $\left(T_{1}\right)$ times of highest energy excitons in V152N and W184F mutants suggest that site energies of BChls 1 and 6, believed to play an important role in receiving excitation from the baseplate BChls, likely play a critical role to ensure the fs energy relaxation observed in wild-type FMO complexes.

\section{References}

1. J. Wen, H. Zhang, M. L. Gross, R. E. Blankenship, Biochemistry, 50, 3502 (2011)

2. A. Khmelnitskiy et al., Biochim. Biophys. Acta, 1859, 165 (2018)

3. E. Thyrhaug et al., J. Phys. Chem. Lett., 7, 1653 (2016) 